\title{
Car drivers' characteristics and the maximum walking distance between parking facility and final destination
}

\author{
Peter van der Waerden \\ Eindhoven University of Technology \\ p.j.h.j.v.d.waerden@tue.nl \\ Marloes de Bruin-Verhoeven \\ Eindhoven University of Technology \\ m.d.bruin.verhoeven@tue.nl
}

\author{
Harry Timmermans \\ Eindhoven University of Technology \\ h.j.p.timmermans@tue.nl
}

\begin{abstract}
In this paper the relationship between car drivers' personal and trip characteristics and the maximum distance car drivers are willing to walk between a parking facility and the final destination(s) will be discussed. The willingness to walk is investigated in the context of four different trip purposes: weekly shopping, non-weekly shopping, work, and social activities. The analyses are based on responses of almost 340 members of the Eindhoven University of Technology's University Parking Panel. The questions regarding car drivers' willingness to walk were included in an Internet-based questionnaire that was distributed in 2011. It appears that car drivers are willing to walk short distances in the case of weekly shopping and work. Longer walking distances are accepted in the case of non-weekly shopping. The influence of car drivers' personal and trip characteristics was investigated using multinomial regression analysis. This analysis shows that the most influential characteristics are the trip-related characteristics "frequency of car use" and "visit duration." The parameter estimates show that in the case of weekly and non-weekly shopping, the more the car is used and the longer car drivers stay at a destination, the higher the probabilities of longer-distance categories. For the trip purposes work and leisure, the opposite holds true.
\end{abstract}

\section{$1 \quad$ Introduction}

Parking facilities may contribute significantly to the attractiveness of shopping environments. Good parking facilities located a reasonable distance from shops may entice people to visit those shops, or the lack of such facilities may prevent them from visiting. In the past, several studies have investigated the effects of walking distance on the attractiveness of parking facilities and, in addition, on the destination, travel mode, and parking-choice behavior of travelers. In general, walking distance is acknowledged as

Copyright 2015 Peter van der Waerden, Harry Timmermans \& Marloes de Bruin-Verhoeven http://dx.doi.org/10.5198/jtlu.2017.568

ISSN: 1938-7849 | Licensed under the Creative Commons Attribution - Noncommercial License 3.0

The Journal of Transport and Land Use is the official journal of the World Society for Transport and Land Use (WSTLUR) and is published and sponsored by the University of Minnesota Center for Transportation Studies. This paper is also published with sponsorship from WSTLUR and the Institutes of Transportation Studies at the University of California, Davis, and University of California, Berkeley. 
one of the most influential parking characteristics (for an extensive overview of research findings regarding the influence of various parking characteristics see Van der Waerden 2012). In line with the finding that walking distance is important, municipalities try to optimize the supply of parking facilities in terms of finding an optimal number of parking spaces and locations for these spaces. It is not clear if municipalities are aware of car driver's preferences regarding the location of parking facilities. In general, it is assumed that short-stay parking facilities (for shopping, medical visits, drop off someone, etc.) have to be located close to the final destination and that long-stay parking facilities (for work, recreation, travel, etc.) can be located at some distance. Figure 1 shows an example of different locations of various types (long and short stay) of parking facilities that are available around King Shaka International Airport, South Africa. Parking facilities for short stays are located close to the entrance of the airport building, while long-stay parking is provided at some distance.

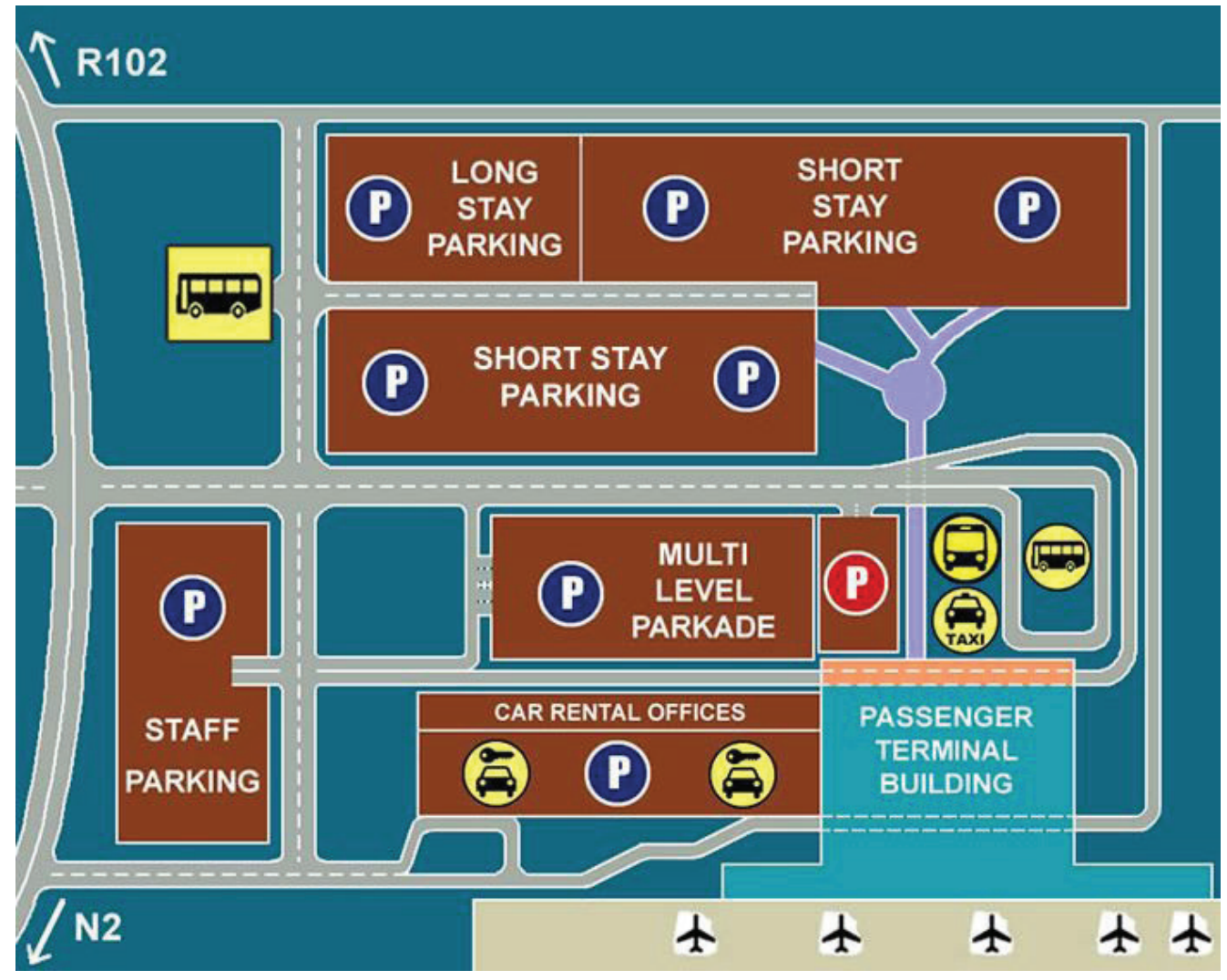

Figure 1: Locations of short- and long-term parking at King Shaka International Airport, South Africa

Most studies regarding maximum walking distances come up with findings in a specific travel context. In many cases, this context is defined in general terms of trip purpose (shopping, commuting, recreational, etc.). For example, Marsden (2006) gives an overview of parking studies in the context of different trip purposes (commuting, commercial and leisure uses, and residential parking). Other triprelated variables such as length of stay at the destination and trip frequency are mostly neglected. The same holds true for the influence of personal characteristics of the car driver. The question is whether these contextual variables (personal and trip characteristics) also influence car drivers' evaluation of walking distance.

Therefore, the aim of this study is to contribute to the limited knowledge concerning maximum 
walking distance by examining the influence of personal and trip characteristics on car drivers' willingness to walk between parking facility and final destination(s).

To achieve this goal, the remainder of this paper is organized as follows. First, attention is paid to previous parking studies that deal with walking distances in different contexts. Next, the adopted research approach is outlined. This section is followed by a brief description of the data collection and sample composition. The analyses are described in the subsequent sections. The paper ends with the conclusions.

\section{$2 \quad$ Walking distance}

In the past, a variety of studies have been carried out regarding the importance of walking distance between parking facility and final destination in relation to travelers' decision process. Walking distance is mostly included in studies regarding car drivers' parking-choice behavior. Only a few studies consider walking distance or time between parking and final destination in relation to travel mode choice (e.g., Beunen, Jaarsma, and Regnerus 2006), route choice (e.g., Shoup 2009; Van Ommeren, Wentink, and Rietveld 2012), parking facility choice (e.g., Benenson, Martens, and Birfir 2008), parking type (e.g., Kobus et al. 2013), and destination choice (e.g., Van der Waerden 2012).

This study focuses on the influence of walking distance in the context of car drivers' parking-choice behavior. Several studies show that walking distance is an important parking attribute when looking at parking choice behavior in the context of shopping behavior (e.g., Bonsall and Palmer 2004; CROW 2003; Guan et al. 2005; Yanjie et al. 2007; Van der Waerden 2012). To illustrate the position of walking distance in relation to other parking attributes Figures 2 and 3 are included. Figure 2 shows that for car drivers, walking distance is the most important factor in the context of parking choice. In the context of the composition of the parking consideration (or choice) set, walking distance is one of the important attributes of parking facilities to be included in the car driver's consideration (or choice) set (Figure 3).

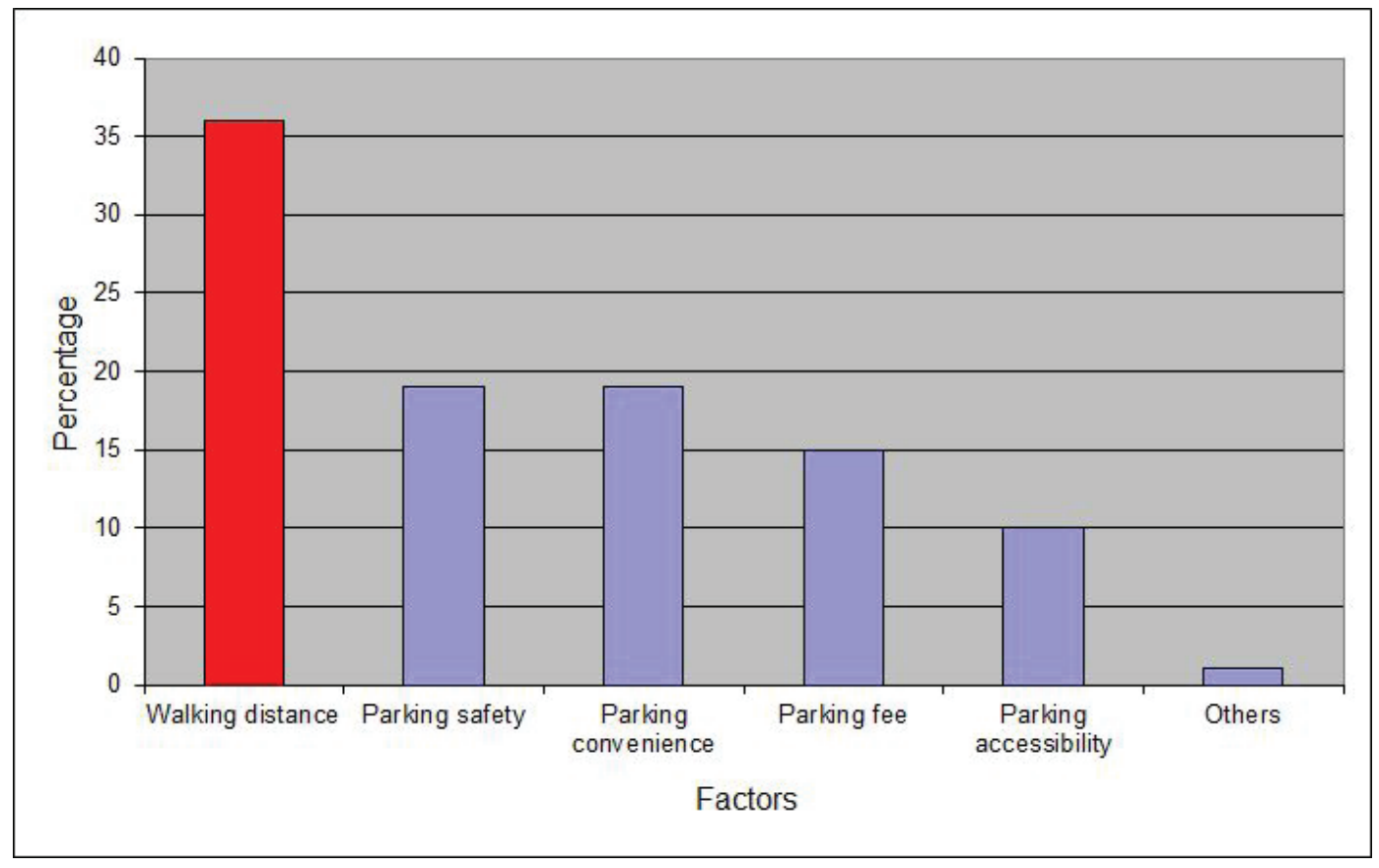

Figure 2: Important factors in the context of parking choice (Yanjie et al. 2007) 


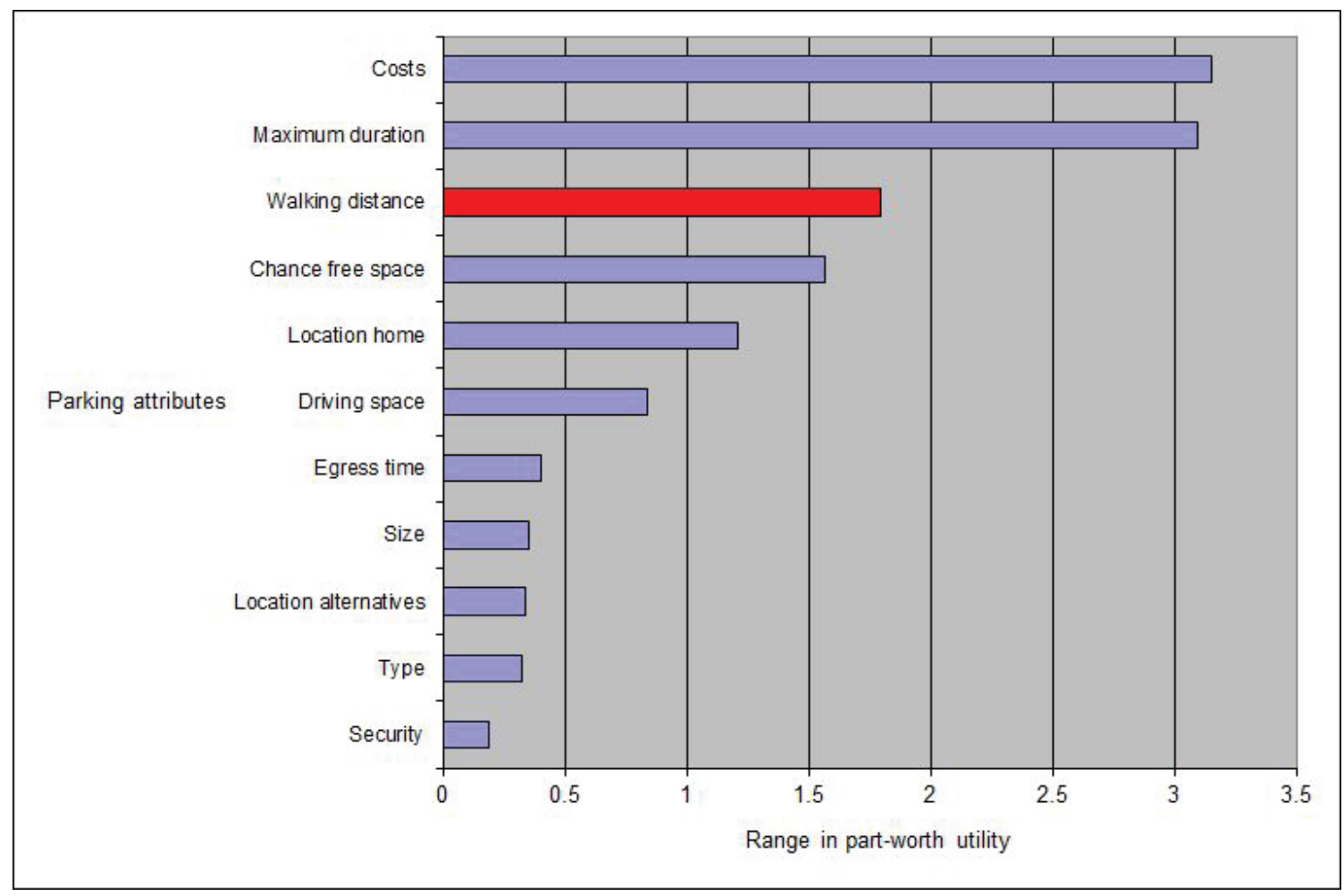

Figure 3: Importance of parking attributes in the context of consideration set composition (Van der Waerden 2012)

CROW (2003) discusses some relationships between maximum walking distance and car drivers' characteristics, namely visit duration and visit purpose, in the context of shopping. Regarding the visit duration, CROW stated that the longer a consumer stays in the shopping center, the longer the walking distance between parking and destinations can be. Consumers who visit the shopping center for nonweekly shopping accept a longer walking distance than consumers who visit a center for weekly (supermarket) shopping. The suggested relations depend on size of the shopping center, the attractiveness of the walking route, and the parking tariff structure.

Also in the parking simulation model ParkAgent in Benenson, Martens, and Birfir (2008), walking distance plays an important role. When modeling car drivers' parking choice behavior, it is assumed that car drivers base their decision to park on three assumptions:

- To find a parking place as close to the destination as possible

- To find a parking place as quickly as possible

- To pay as little as possible

Benenson, Martens, and Birfir (2008) characterized four groups of drivers: residents, guests, employees, and customers. The groups mostly differ regarding destination, arrival time, and duration of parking. No detailed information regarding preferred walking distances per group of drivers is available.

The importance of walking distances in relation to car drivers' parking choice behavior is less clear in other contexts than shopping (as presented above). For example, Borgers et al. (2010) found that residents consider walking distance to be less important than the presence of motorized traffic and security in the context of residential parking. Van der Waerden, Timmermans, and Vermeulen (2013) found that residents consider paid parking as the most important attribute when evaluating different parking situations in residential areas, followed, at some distance, by walking distance between residence and parking.

In contrast to previous studies, this study focuses on individuals' preferences regarding the maxi- 
mum walking distance instead of optimal distance. This approach is especially interesting because it is often not possible to locate parking facilities close to travelers' destinations. In addition, this study covers different trip purposes at the same time. Respondents will be asked to indicate the maximum walking distance for weekly shopping, non-weekly shopping, work trips, and social trips at the same time. The main benefit of this approach is that respondents can compare the distances for different trip purposes.

\section{Research approach}

To gain insight into the influence of contextual characteristics on car drivers' evaluation of walking distances, the following research approach was adopted. First, various levels of walking distance between parking facility and final destination were defined, based on experiences in previous studies. Six different distance categories were defined covering the distance between approximately 50 and 700 meters (e.g., CROW 2003 for shopping context; Benenson, Martens, and Birfir 2008 for residential context):

- Less than or equal to 50 meters

- Approximately 100 meters

- Approximately 200 meters

- Approximately 300 meters

- Approximately 400 meters

- 500 meters or more

Next, the question regarding car drivers' evaluation of maximum walking distance was formulated and included in an extensive Internet-based questionnaire regarding car drivers' parking preferences in the context of different trip purposes. The exact formulation of the question was: What maximum distance are you willing to walk for...?

The Internet-based questionnaire was set up in a non-commercial questionnaire system developed by the Eindhoven University of Technology. The questionnaire focused on four different trip purposes: weekly shopping, non-weekly shopping, working, and social activities. Weekly shopping concerns shopping for daily products in, for example, a supermarket, bakery, or butcher shop. Non-weekly shopping covers the purchase of shoes, clothing, etc. For each type of trip, some background information (usual time of stay and visit frequency) was also collected. The respondents' personal characteristics were collected at the moment that respondents signed up for the University Parking Panel. The current questionnaire included four different personal characteristics: gender, age, educational level (also included as representative of income level), and home location. The effects of the contextual characteristics on the car drivers' evaluation were investigated using multinomial logistic regression analysis.

The data used to conduct the regression analysis were collected using the University Parking Panel (see www.parkeerpanel.nl). This panel is an initiative of the Eindhoven University of Technology and Empaction BV and was started in 2009. The aim of the panel is to create a platform of people who are willing to participate in different parking-related studies as a common car driver. The panel is consulted approximately four times per year for different subjects such as introduction of paid parking in residential areas, car drivers' willingness to use Park and Ride, and car drivers' familiarity with the city's parking situation. All results of the consultations are presented online. Participants of various travel-related studies of the university were invited to become a member of the panel. This group was extended by means of personal invitation to family, colleagues, and acquaintances. The panel was also promoted in Dutch journals, through LinkedIn, at meetings, and on different web pages.

At the moment of data collection for this study, the panel consisted of 632 participants. The total panel is not yet representative of the Dutch population, but it consists of a usable mixture of individuals (many types of individuals are included in the panel). In May 2011, all participants of the panel were invited to fill out an Internet-based questionnaire regarding car drivers' parking preferences. In total, 336 
respondents (53.2 percent) completed the relevant questions at the moment of the analyses. The details of the respondents are presented in Table 1 . The table shows that more males than females participated, and that the number of more highly educated individuals is larger than the number of medium-educated individuals. This distribution is in line with the distribution across the total panel.

Table 1: Characteristics of respondents

\begin{tabular}{|l|l|c|c|c|}
\hline \multicolumn{1}{|c|}{ Characteristic } & \multicolumn{1}{|c|}{ Level } & Frequency & Percentage & Coding* \\
\hline \multirow{2}{*}{ Gender } & Male & 222 & 66.1 & +1 \\
\cline { 2 - 5 } & Female & 114 & 33.9 & -1 \\
\hline \multirow{3}{*}{ Age } & $\begin{array}{l}\text { Younger than 50 years } \\
\text { of age }\end{array}$ & 180 & 53.6 & +1 \\
\cline { 2 - 5 } & 50 years of age and older & 156 & 46.4 & -1 \\
\hline \multirow{2}{*}{ Educational level } & Medium & 109 & 32.4 & +1 \\
\cline { 2 - 5 } & High & 227 & 67.6 & -1 \\
\hline \multirow{2}{*}{ Residential location } & Eindhoven & 216 & 64.3 & +1 \\
\cline { 2 - 5 } & Outside Eindhoven & 120 & 35.7 & -1 \\
\hline \multicolumn{2}{|l|}{ Number of respondents and total percentage } & 336 & 100.0 & \\
\hline
\end{tabular}

* coding used in the regression analyses

Table 2 presents an overview of the experiences respondents have with the use of cars for each trip purpose. It appears that most respondents have experience with the use of the car for all kinds of trips. The numbers for non-weekly shopping and working trips are lower but still usable. The lower number for this trip purpose could be expected in the Netherlands where the bike and public transport are popular means of transport. This holds true especially for non-weekly and commuting trips.

Table 2: Experiences with car trips per trip purpose

\begin{tabular}{|l|c|c|c|}
\hline \multicolumn{1}{|c|}{ Trip purpose } & Level & Frequency & Percentage \\
\hline \multirow{2}{*}{ Weekly shopping } & Yes & 300 & 89.3 \\
\cline { 2 - 4 } & No & 36 & 10.7 \\
\hline \multirow{2}{*}{ Non-weekly shopping } & Yes & 246 & 73.2 \\
\cline { 2 - 4 } & No & 90 & 26.8 \\
\hline \multirow{2}{*}{ Work } & Yes & 204 & 60.7 \\
\cline { 2 - 4 } & No & 132 & 39.3 \\
\hline \multirow{2}{*}{ Social visits } & Yes & 295 & 87.8 \\
\cline { 2 - 4 } & No & 41 & 12.2 \\
\hline Total & & 336 & 100.0 \\
\hline
\end{tabular}

Respondents who indicated that they have experience with a certain trip purpose, were asked to indicate how many times they use the car (frequency) and how long they stay at a destination (duration). Table 3 presents for each trip purpose the visit frequency and visit duration. For both categorical attributes (measured at ordinal level), two attribute levels are defined. In the case of frequency, the level "frequent" includes almost daily and at least once per week, while the level "infrequent" includes a few times per month and a few times per year. The levels of duration are separately specified for each trip purpose. For all trip purposes, the level "short" includes visits of less than or equal to one hour, and the level "long" includes visits of more than one hour. 
Table 3: Characteristics of trips

\begin{tabular}{|c|c|c|c|c|c|}
\hline \multirow[b]{2}{*}{ Attributes } & \multirow[b]{2}{*}{ Levels (coding) } & \multicolumn{4}{|c|}{ Trip purpose } \\
\hline & & $\begin{array}{l}\text { Weekly } \\
\text { shop- } \\
\text { ping }\end{array}$ & $\begin{array}{c}\text { Non- } \\
\text { weekly } \\
\text { shopping } \\
\end{array}$ & work & Social visits \\
\hline \multirow{2}{*}{ Frequency } & Frequent & 79.3 & 13.0 & 88.2 & 28.1 \\
\hline & $(-1)$ & 20.7 & 87.0 & 11.8 & 71.9 \\
\hline \multirow{4}{*}{ Duration } & Infrequent & 80.0 & 7.7 & 1.0 & 3.1 \\
\hline & $(+1)$ & 20.0 & 92.3 & 99.0 & 96.9 \\
\hline & Short (-1) & & & & \\
\hline & Long $(+1)$ & & & & \\
\hline \multicolumn{2}{|l|}{$\mathrm{N}$} & 300 & 246 & 204 & 295 \\
\hline
\end{tabular}

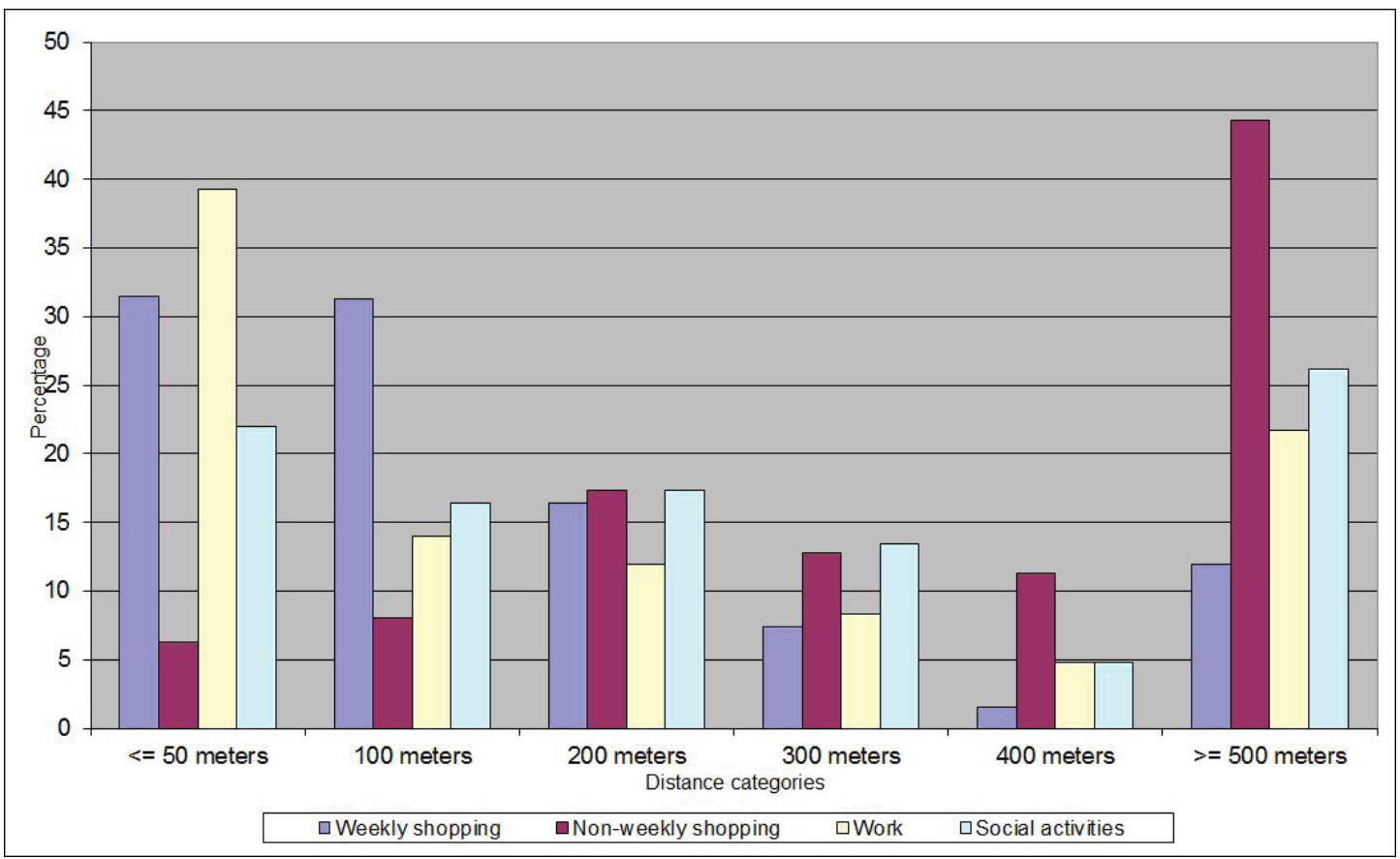

Figure 4: Maximum distance car drivers are willing to walk per trip purpose

\section{$4 \quad$ Analyses}

In the first part of the analyses, attention is paid to car drivers' evaluation of the walking distances (Figure 4). As expected, the maximum distance car drivers are willing to walk is short in the context of weekly shopping and work and long in the context of non-weekly shopping. For social activities, the maximum distance car drivers are willing to walk is more spread out over the distance classes.

The relationship between the maximum distance car drivers are willing to walk (dependent variable) and personal and trip characteristics (independent variables) is investigated using multinomial regression analyses. The formula of the multinomial regression model is defined as follows: 


$$
\begin{array}{ll}
\text { In }\left(\frac{\left.P \text { alternative }_{k}\right)}{P(\text { reference })}\right)=b_{k 0}+\sum_{1} b_{k 1} \cdot X_{l} \\
\text { where, } & \text { is choice alternative } k ; \\
P\left(\text { alternative }_{k}\right) & \text { is the reference alternative; } \\
\mathrm{P}(\text { reference })^{2} & \text { is alternative specific constant of alternative } k ; \\
b_{k 0} & \text { is parameter for alternative } k \text { and attribute } l ; \\
b_{k l} & \text { is value of attribute } l ;
\end{array}
$$

Before starting the regression analyses, a correlation matrix was created to investigate the correlations between all included independent variables. It appeared that there were no high correlations between included variables. Based on this finding, all independent variables were included in the regression analyses.

As described before, the dependent variable is subdivided into six categories (see section Research Approach). To find an optimal model, models with different numbers (3, 4, 5, and 6) of distance categories were tested. It appeared that the most optimal model could be built for three distance categories: category one (less than or equal to 100 meters); category two (between 100 and 300 meters); and category three (more than 300 meters). The first category is used as a reference category. Effect coding (see Tables 2 and 3) is used to represent the contribution of the personal and trip characteristics to the utility of the categories. The results of the multinomial regression analyses are presented in Table 4 . The model fitting information shows that all models perform quite well with the exception of the model for social activities. Given the degrees of freedom (12), the test values represented by the Chi-square test $\left(-2^{*}[\log\right.$ likelihood "null" model minus Log-likelihood "optimal" model]) are significant for all models, at least at the 90-percent confidence level.

The values in Table 4 show the influence of the attributes on the probability that an alternative is chosen in relation to the reference category. In this case, the category "100 meters or less" is used as a reference. The parameter estimate of the attribute home location $(-0.325)$ shows that the probability of distance category two increases compared to the first category when a respondent lives in the suburbs. For this attribute (home location) the same effect is found for the third distance category. In general, the parameter estimates show the following effects. In the context of weekly shopping trips, the probabilities of distance categories two and three increase when the respondent has a medium-education level (lower/ medium income level), lives in the suburbs, uses the car infrequently, and/or stays for a longer period at the destination. Looking to non-weekly shopping trips, the probabilities of distance categories two and three increase when the respondent has a high-education level, uses the car infrequently, and/or stays for a longer period at the destination. Now, no effect is found for the resident's home location.

In the case of work trips, only three parameters are significant. The effects of the parameters of education and frequency are similar to the effects found for weekly and non-weekly shopping. The effect of duration is different from previous findings. Now, the parameter indicates that the probability of distance category two decreases (instead of increases like before) when the respondents stay for a longer period. This means that respondents prefer a short walking distance (100 meters or less) in the case of a long stay at the destination. In contrast to shoppers, workers want to park close to their destination even when they stay for a longer period.

Finally, the effect of education in the context of social activity trips is similar to the effect found before. Similar to work trips, the effect of duration differs from previous findings. The parameter shows that the probability of distance category three decreases when a respondent stays for a longer period at 
the destination. This means that respondents prefer a parking facility close to the destination when they stay for a longer period.

Table 4: Model estimation results of multinomial regression analyses

\begin{tabular}{|c|c|c|c|c|c|}
\hline \multirow[b]{2}{*}{ Distance Category $_{1}$} & \multirow[b]{2}{*}{ Characteristics } & \multicolumn{4}{|c|}{ Trip purposes } \\
\hline & & Weekly Shopping & $\begin{array}{c}\text { Non-weekly } \\
\text { shopping }\end{array}$ & Work & Social activities \\
\hline \multirow{6}{*}{$\begin{array}{l}\text { Category 2: Between } \\
100 \text { and } 300 \text { meters }\end{array}$} & Gender & -0.055 & -0.244 & -0.066 & -0.113 \\
\hline & Age & -0.246 & 0.262 & -0.036 & -0.075 \\
\hline & Education & -0.084 & -0.748 & -0.436 & -0.305 \\
\hline & Location & -0.325 & -0.211 & -0.103 & -0.272 \\
\hline & Frequency & 0.396 & 0.750 & 0.157 & 0.127 \\
\hline & Duration & 0.548 & 0.384 & -0.885 & -0.259 \\
\hline \multirow{6}{*}{$\begin{array}{l}\text { Category 3: More than } \\
300 \text { meters }\end{array}$} & Gender & 0.099 & -0.171 & 0.421 & -0.067 \\
\hline & Age & -0.225 & 0.184 & 0.222 & -0.139 \\
\hline & Education & 0.391 & -0.703 & -0.211 & -0.331 \\
\hline & Location & -0.370 & -0.129 & -0.278 & -0.142 \\
\hline & Frequency & 0.667 & 0.714 & 0.867 & 0.069 \\
\hline & Duration & 0.899 & 0.827 & -0.473 & -0.442 \\
\hline \multicolumn{6}{|l|}{ Model fitting information } \\
\hline \multicolumn{2}{|l|}{ Chi-square } & 107.77 & 79.013 & 56.374 & 18.835 \\
\hline \multicolumn{2}{|c|}{ Sign. Likelihood Ratio Statistic } & 0.000 & 0.000 & 0.000 & 0.093 \\
\hline
\end{tabular}

${ }^{1}$ Reference category is 100 meters or less

${ }^{2}$ Bold indicates significant at conventional (95 percent) level

\section{Conclusions}

This paper intends to give more insights into differences in preferred walking distances between different groups of car drivers. Four different trip purposes are distinguished: weekly shopping trips, non-weekly shopping trips, work trips, and trips to conduct social activities. The descriptive analyses show clear differences between car drivers' preferences for various trip purposes. The maximum distance car drivers are willing to walk is short in the context of weekly shopping (approximately 100 meters) and work (maximum 50 meters), and long in the context of non-weekly shopping (500 meters or more). The relationship between preferred maximum walking distances and various personal and trip characteristics is investigated using multinomial regression analyses. It appears that the most influential characteristics are the trip-related characteristics frequency of car use and visit duration. The parameter estimates show that the more the car is used and the longer car drivers stay at a destination, the higher the probabilities are of longer distance categories.

The results of this study can give support to planners who intend to reorganize their parking supply in the vicinity of inner city areas in favor of a more optimal use of individual parking facilities and, in addition, a more efficient use of the urban transportation network. The parking regime of individual parking facilities can now be adapted to the most likely users based on the location of the facilities visà-vis destinations.

The current study included some limitations that have to be investigated in more detail in the future. The following research topics can be considered as relevant in this respect. A considerable deficiency of the study concerns the size and the composition of the sample. The sample does not fully represent the population that is involved in the investigated trip purposes. A more stratified sampling 
procedure that fits better to the sub-populations is necessary to get a more optimal sample. For example, respondents evaluating the maximum walking distance for weekly and non-weekly shopping could be recruited in shopping centers.

Also some improvements regarding some detailing of characteristics could be included in future research. For example, the level of individuals' walking ability is not included in this study. The increase of the number of older people and the increased participation of challenged people in common life make this topic relevant. In this study, for all personal and trip characteristics only two levels are distinguished. A subdivision into more levels (covering more different groups of individuals and showing non-linear relations) could increase required insights regarding the maximum distances car drivers are willing to walk between a parking facility and final destination(s). Finally, maximum distance is defined in a very general way. More detailed specifications could be considered such as walking distance to specific destinations or activities (e.g., location where goods have to be carried to/from). Also a more detailed subdivision of the distances classes can be considered (e.g., classes of 25 or 50 meters instead of 100 meters). 


\section{References}

Benenson, I., K. Martens, and S. Birfir. 2008. ParkAgent: An agent-based model of parking in the city. Computers, Environment and Urban Systems 32(6): 431-439.

Beunen, R., C. F. Jaarsma, and H. D. Regnerus. 2006. Evaluating the effects of parking policy measures in nature areas. Journal of Transport Geography 14(5): 376-383.

Bonsall, P., and I. Palmer. 2004. Modeling drivers' car parking behavior using data from a travel choice simulator.Transportation Research C 12(5): 321-347.

Borgers, A., A. Kemperman, L. Toll, and H. Timmermans. 2010. Measuring preferences for parking facilities in old residential areas. Proceedings of the 7th International Symposium on City Planning and Environmental Management in Asian Countries, January 9-12, Fukuoka, Japan.

CROW. 2003. Walking distances in shopping areas (in Dutch). From Parking Management to Mobility Management, Part 7. Eide, the Netherlands: Center for Research and Contract Standardization in Civil and Traffic Engineering.

Guan, H., X. Sun, X. Liu, and L. Liu. 2005. Modeling parking behavior of better control and pricing: A case study from one of the busiest retail shopping areas in Beijing, China. Compendium of Papers of the 84th Annual Meeting of the Transportation Research Board, January 9-13, Washington DC.

Kobus, M. B. W., E. Gutiérrez-i-Puigarnau, P. Rietveld, and J. N. van Ommeren. 2013. The on-street parking premium and car drivers' choice between street and garage parking. Regional Science and Urban Economics 43(2): 395-403.

Marsden, G. 2006. The evidence base for parking policy—A review. Transport Policy 13(6): 447-457.

Shoup, D. C. 2006. Cruising for parking. Transport Policy 13(6): 479-486.

Van Ommeren, J. N., D. Wentink, and P. Rietveld. 2012. Empirical evidence on cruising for parking. Transport Research Part A 46(1): 123-130.

Van der Waerden, P. 2012. PAMELA: Parking analysis model for predicting effects in local areas. Thesis. Eindhoven, the Netherlands: Eindhoven University of Technology.

Van der Waerden, P., H. Timmermans, and H. Vermeulen. 2013. Parking in residential areas: A stated choice analyses of residents' preference. Proceedings of the 13th World Conference on Transport Research, July 15-18, Rio de Janeiro, Brazil.

Yanjie J. D., W. Deng, W. Wang, and G. Liu. 2007. Two-phased parking choice model for pre-trip parking guidance system. Compendium of Papers of the 86th Annual Meeting of the Transportation Research Board, January 21-27, Washington DC. 